\title{
Prolonged Release of the Local Anesthetic Butamben for Potential Use in Pain Management
}

\author{
Ashish Rastogi ${ }^{1,2}$, Salomon Stavchansky ${ }^{2}$, Phillip D. Bowman ${ }^{{ }^{*}}$ \\ ${ }^{1}$ US Army Institute of Surgical Research, Fort Sam Houston, USA; ${ }^{2}$ Division of Pharmaceutics, College of Pharmacy, The Univer- \\ sity of Texas at Austin, Austin, USA. \\ Email: stavchansky@mail.utexas.edu, ’phillip.bowman@us.army.mil
}

Received March 20 ${ }^{\text {th }}$, 2012; revised April 29 ${ }^{\text {th }}$, 2012; accepted May $11^{\text {th }}, 2012$

\begin{abstract}
Continuous delivery of local anesthetics might be useful for management of localized and chronic pain. Controlled release injectable anesthetics have been developed but they can deliver the drug for only few days and the release is not zero-order. A drug delivery system (DDS) consisting of a perforated reservoir for drug containment and release and its potential for management of chronic pain is described. Proof of principle is detailed for long-term zero order delivery of butamben. In this study, the DDS was a polyimide tube with a $0.20 \mathrm{~mm}$ hole and butamben release was evaluated in vitro. It is envisioned that the DDS could be implanted in proximity to a nerve, enervating the pain source, for long-term control of chronic pain.
\end{abstract}

Keywords: Controlled Release; Prolonged Drug Delivery; Pain Management; Butamben

\section{Introduction}

Long-term drug therapy for pain management is challenging and often requires multiple dosing with incremental increases in the dose [1]. Frequent high-dose regimens may initiate complications such as drug abuse, drug dependence, addiction, hypotension, respiratory depression, and mental confusion [2,3]. Additionally, frequent systemic dosing may cause patients distress and interfere with their day-to-day activities.

Although local anesthetics have been used to provide local analgesia, these formulations suffer from inadequate duration of action [4]. Some of these concerns have been addressed by development of targeted delivery systems based on liposomes that result in lower dosing frequencies, higher efficacy and lower side effects. However these systems can only produce the desired effect for few days and rely on other drugs such as glucocortocoids for extending the duration of nerve block $[4,5]$. Intrathecal pumps and controlled release injectable formulations are another pain management methods that can deliver constant amounts of drug to the targeted area producing therapeutic effects at a considerable lower dose [6,7]. For example, an intrathecal morphine pump can deliver the drug long-term directly to the spinal fluid at much lower quantities than would be required by an oral dose [8]. However, intrathecal pumps suffer from their own limitations because they are bulky, battery-

"Corresponding author. operated, and not suitable for everyone [9]. Persistent cerebrospinal fluid leak during its insertion is also a known complication [10].

The present investigation reports on the application of a drug delivery system capable of delivering drugs at a constant rate [11,12]. This drug delivery system could be used for controlling localized pain for long periods of time. This delivery system is practical and offers a safer alternative to the systemic use of analgesics. It is an implantable system capable of zero order and long-term targeted delivery of a drug with appropriate solubility. Butamben ( $n$-butyl-p-aminobenzoate) was selected as the drug of study because of its low water solubility. A poorly soluble drug will ensure slow and long-term release without use of any excipients. Butamben is a long-acting local anesthetic similar to benzocaine and is used for the treatment of chronic pain when given as an epidural suspension $[13,14]$.

\section{Methods}

\subsection{Drug Loading}

Polyimide tubing (inside diameter $=1.8 \mathrm{~mm}$, Microlumen, FL, USA) was cut to $20 \mathrm{~mm}$ in length. A $0.2 \mathrm{~mm}$ hole made with a titanium wire was placed at the center of the tube. The perforated tubes (Figure 1) were packed aseptically with dry butamben powder (Sigma Aldrich, MO, USA) and the ends of the tube sealed with heat 


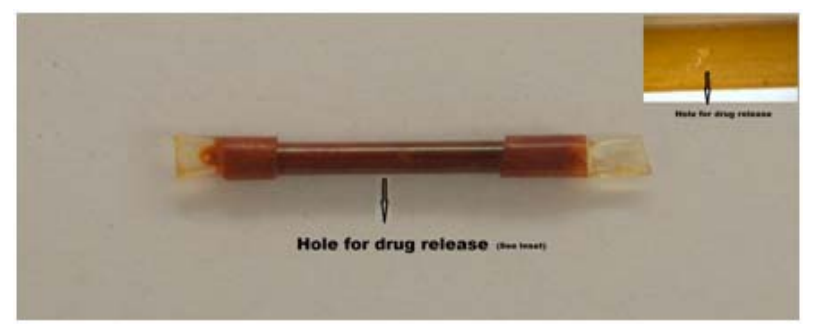

Figure 1. The drug delivery system with microhole on the surface. Hole diameter $=200 \mathrm{~mm}$; Tube diameter $=1.8 \mathrm{~mm}$; Tube length $=20 \mathrm{~mm}$. The size of the device and the perforations can be scaled to fit the need of the therapeutic application [12].

shrink polyolefin tubing.

\subsection{In Vitro Drug Release Studies}

Twelve tubes were loaded. Each tube was placed in a microvial containing $1.8 \mathrm{ml}$ of phosphate-buffered saline (PBS). Non-perforated polyimide tubes loaded with butamben were used as control in the study. The microvials were placed on a rocker (46 - 48 oscillations $/ \mathrm{min}$ ) and maintained inside an incubator $\left(37.0^{\circ} \mathrm{C} \pm 1.0^{\circ} \mathrm{C}\right)$ for the entire duration of study. Aliquots were collected at regular intervals and replenished with fresh PBS. The collected samples were analyzed spectro-photometrically at $280 \mathrm{~nm}$ to estimate the amount of butamben released.

\subsection{Statistical Analysis}

Levene's test was used to access the homogeneity of variance in various groups. One-way analysis of variance (ANOVA) with post hoc analysis using Tukey's honestly significant difference (HSD) test (equal variance assumed) or Games-Howell test (equal variances not assumed) through SPSS statistical software was used to analyze differences among the subsets with respect to hole size and drug loading. Linear regression analysis of the cumulative release data and F-statistics was used to estimate the amount of butamben released as a function of time. The significance level of the study was set at $P<0.05$.

\section{Results}

An average amount of $34.9 \pm 1.6 \mathrm{mg}$ of butamben was loaded into the device. Butamben delivery from the microtubes was monitored in vitro for 124 days ( 4 months) and is illustrated in Figure 2. The release rate was zero order with an $\mathrm{R}^{2}$ value of 0.9992 . The linearity of the release was further confirmed by F test, F $(1,56), P<$ 0.05 . An average of $19.4 \pm 3.7$ micrograms of butamben was released per day. Assuming a constant rate of release of butamben, a period longer than 1 year is achievable based on the data. In comparison, a 5\% - 10\% suspension of butamben in volumes between 15 and $25 \mathrm{ml}$ for

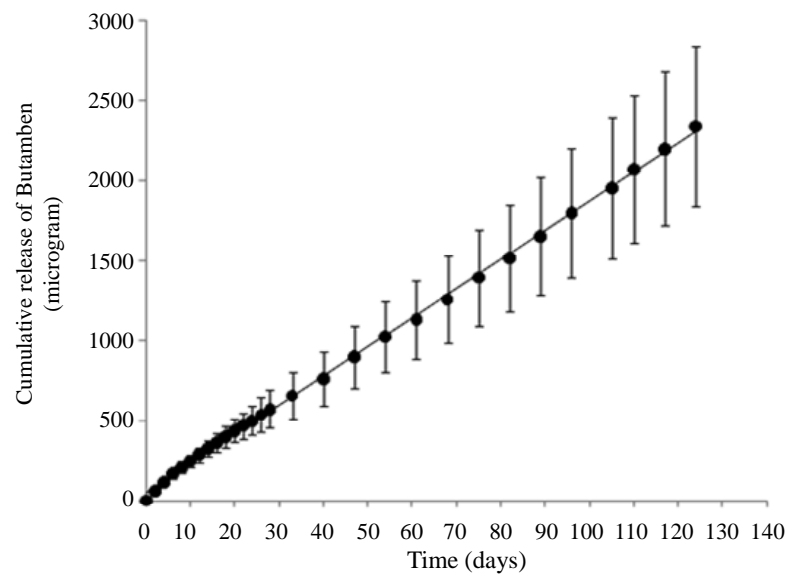

Figure 2. A constant amount of butamben was released from the perforated tubes. The solid line represents the regression line. The slope of the line is the rate of drug release per day. The data is represented as mean with standard deviation, $\mathbf{n}=12$.

epidural injections and 5 and $20 \mathrm{ml}$ for peripheral nerve blocks is administered to treat chronic pain [14].

\section{Discussion}

Pain is triggered in the nervous system by injury or disease and is often known as the fifth vital sign [15]. Although potent analgesics are administered to treat acute pain, management of chronic pain such as in a spinal injury or phantom limb pain requires long term pharmacological and non-pharmacological intervention ranging from several months to years [16-18]. In 2010, the Department of Health and Human Services enlisted Institute of Medicine (IOM) of the National Academies to examine pain as a public health problem. According to the report published by IOM, chronic pain affects more than 100 million American adults, which is more than the combined number of patients affected by heart diseases, cancer, and diabetes [19]. The medical treatment and lost productivity due to pain results in a loss of up to $\$ 635$ billion each year to the nation.

The DDS investigated in this study is an implantable system capable of long term controlled release of drugs. As suggested by the in vitro study, a prolonged release of local anesthetic can be achieved for several months. Hence, it may be possible to decrease the health care cost associated with long term drug therapy as frequent dosing and visit to health care clinics can be avoided. Further in vivo studies are required to establish butambens' and the DDSs' efficacy in comparison to the 5\% $10 \%$ suspension. However, the desired drug release rate can be obtained by varying different parameters of the DDS such as tube size, hole size, and number of holes on the tube. The underlying mechanism of drug release from the DDS is simple diffusion. Diffusion-controlled re- 
servoir-type DDSs' are known to yield zero order drug kinetics (i.e. delivery at a constant rate) as long as the concentration gradient is maintained constant [20]. A constant concentration gradient may be achieved by having a large volume of drug in the donor side (reservoir) and a sink condition in the receptor side (exterior). Solid drug presents the maximum amount of drug that can be contained within the donor compartment. In the present study, dry butamben powder was packed to achieve a high concentration gradient. Preliminary in vitro data suggests that the DDS is capable of producing long-term zero order kinetics. The novelty of the device is its small size and potential ability to deliver butamben for more than a year. Another advantage of the DDS may be that the drug is protected inside the device from the hostile physiological environment and do not require any other excipients or active ingredients to prolong the release rates.

Additionally, unlike other pain management implants, the DDS is not battery-operated and can be placed at various anatomical sites. It should be possible to use such a device for local and controlled delivery of drugs for management of chronic pain. Although a nonbioabsorbable material (polyimide) was used in this study, the technique could be extended to a bioabsorbable material as long as the degradation rate of the material is slower than the dissolution rate of the drug. The DDS can be implanted at sensitive sites such as intrathecal space or in the vicinity of a nerve. Further in vivo studies are required to establish the efficacy of the DDS in combination with butamben as an effective pain management tool.

\section{REFERENCES}

[1] J. P. Ney, M. Difazio, A. Sichani, W. Monacci, L. Foster and B. Jabbari, “Treatment of Chronic Low Back Pain with Successive Injections of Botulinum Toxin a over 6 Months: A Prospective Trial of 60 Patients,” The Clinical Journal of Pain, Vol. 22, No. 4, 2006, pp. 363-369. doi:10.1097/01.ajp.0000174267.06993.3f

[2] M. E. Clark, M. J. Bair, C. C. Buckenmaier 3rd, R. J. Gironda and R. L. Walker, "Pain and Combat Injuries in Soldiers Returning from Operations Enduring Freedom and Iraqi Freedom: Implications for Research and Practice," Journal of Rehabilitation Research and Development, Vol. 44, No. 2, 2007, pp. 179-194. doi:10.1682/JRRD.2006.05.0057

[3] K. Miotto, P. Compton, W. Ling and M. Conolly, "Diagnosing Addictive Disease in Chronic Pain Patients," Psychosomatics, Vol. 37, No. 3, 1996, pp. 223-235. doi:10.1016/S0033-3182(96)71561-X

[4] H. Epstein-Barash, I. Shichor, A. H. Kwon, S. Hall, M. W. Lawlor, R. Langer and D. S. Kohane, "Prolonged Duration Local Anesthesia with Minimal Toxicity,” Proceedings of the National Academy of Sciences of the United
States of America, Vol. 106, No. 17, 2009, pp. 7125-7130. doi:10.1073/pnas.0900598106

[5] G. Colombo, R. Padera, R. Langer and D. S. Kohane, "Prolonged Duration Local Anesthesia with Lipid-Protein-Sugar Particles Containing Bupivacaine and Dexamethasone,” Journal of Biomedical Materials Research Part A, Vol. 75, No. 2, 2005, pp. 458-464. doi:10.1002/jbm.a.30443

[6] A. D. Beaulieu, P. M. Peloso, B. Haraoui, W. Bensen, G. Thomson, J. Wade, P. Quigley, et al., "Once-Daily, Controlled-Release Tramadol and Sustained-Release Diclofenac Relieve Chronic Pain Due to Osteoarthritis: A Randomized Controlled Trial," Pain Research \& Management, Vol. 13, No. 2, 2008, pp. 103-110.

[7] H. S. Gilmer-Hill, J. E. Boggan, K. A. Smith and F. C. Wagner Jr., "Intrathecal Morphine Delivered via Subcutaneous Pump for Intractable Cancer Pain: A Review of the Literature,” Surgical Neurology, Vol. 51, No. 1, 1999, pp. 12-15. doi:10.1016/S0090-3019(98)00080-9

[8] O. T. Guttman, M. A. Rosenblatt and T. Mims, "Median Arcuate Ligament Syndrome-A Novel Treatment Using an Intrathecal Morphine Pump to Relieve Intractable Visceral Pain,” Pain Practice, Vol. 8, No. 2, 2008, pp. 133-137. doi:10.1111/j.1533-2500.2007.00166.X

[9] S. Hong, J. S. Lee, J. W. Park, K. Nam, J. Choi, J. C. Lee, J. K. Park, et al., "Development of an Implantable Intrathecal Drug Infusion Pump,” Conference Proceedings of the IEEE-Engineering in Medicine and Biology Society, Vol. 5, 2004, pp. 3440-3442.

doi:10.1109/IEMBS.2004.1403965

[10] J. Worner, K. Kothbauer and H. Gerber, "Intrathecal Morphine Pump Malfunction Due to Leakage at the Catheter Connection Site: A Rare Problem and Its Prevention,” An esthesia \& Analgesia, Vol. 108, No. 6, 2009, pp. 1994-1995. doi:10.1213/ane.0b013e3181a280e4

[11] A. Rastogi, Z. Luo, Z. Wu, P. S. Ho, P. D. Bowman and S. Stavchansky, "Development and Characterization of a Scalable Microperforated Device Capable of Long-Term Zero Order Drug Release,” Biomedical Microdevices, Vol. 12, No. 5, 2010, pp. 915-921. doi:10.1007/s10544-010-9446-X

[12] A. Rastogi, P. Bowman and S. Stavchansky, "Evaluation of a Perforated Drug Delivery System in Mice for Prolonged and Constant Release of a Hydrophilic Drug," Drug Delivery and Translational Research, Vol. 2, No. 2, 2012, pp. 106-111. doi:10.1007/s13346-012-0062-6

[13] L. J. Rampaart, J. P. Beekwilder, G. T. van Kempen, R. J. van den Berg and D. L. Ypey, "The Local Anesthetic Butamben Inhibits Total and L-Type Barium Currents in PC12 Cells,” Anesthesia \& Analgesia, Vol. 106, No. 6, 2008, pp. 1778-1783. doi:10.1213/ane.0b013e318172c3a2

[14] M. Shulman, T. R. Lubenow, H. A. Nath, W. Blazek, R. J. McCarthy and A. D. Ivankovich, "Nerve Blocks with 5\% Butamben Suspension for the Treatment of Chronic Pain Syndromes," Regional Anesthesia and Pain Medicine, Vol. 23, No. 4, 1998, pp. 395-401. doi:10.1016/S1098-7339(98)90014-1

[15] S. M. Fishman, "Pain as the Fifth Vital Sign: How Can I 
Tell When Back Pain Is Serious,” Journal of Pain \& Palliative Care Pharmacotherapy, Vol. 19, No. 4, 2005, pp. 77-79. doi:10.1080/J354v19n04_13

[16] D. J. Gillis, M. Wouda and N. Hjeltnes, "Non-Pharmacological Management of Orthostatic Hypotension after Spinal Cord Injury: A Critical Review of the Literature,” Spinal Cord, Vol. 46, No. 10, 2008, pp. 652-659. doi:10.1038/sc.2008.48

[17] M. A. Hanley, M. P. Jensen, D. M. Ehde, A. J. Hoffman, D. R. Patterson and L. R. Robinson, "Psychosocial Predictors of Long-Term Adjustment to Lower-Limb Amputation and Phantom Limb Pain," Disability and Rehabilitation, Vol. 26, No. 14-15, 2004, pp. 882-893. doi:10.1080/09638280410001708896
[18] C. Mercier and A. Sirigu, "Training with Virtual Visual Feedback to Alleviate Phantom Limb Pain,” Neurorehabilitation and Neural Repair, Vol. 23, No. 6, 2009, pp. 587-594. doi:10.1177/1545968308328717

[19] Institute of Medicine of the National Academies, "Relieving Pain in America-A Blueprint for Transforming, Prevention, Care, Education, and Research,” 2011. http://www.iom.edu/Reports/2011/Relieving-Pain-in-Am erica-A-Blueprint-for-Transforming-Prevention-CareEducation-Research/Report-Brief.aspx

[20] L. Simon, P. Bolisetty and M. N. Erazo, "Dynamics of Dissolution and Diffusion-Controlled Drug Release Systems," Current Drug Delivery, Vol. 8, No. 2, 2011, pp. 144-151. doi:10.2174/156720111794479916 